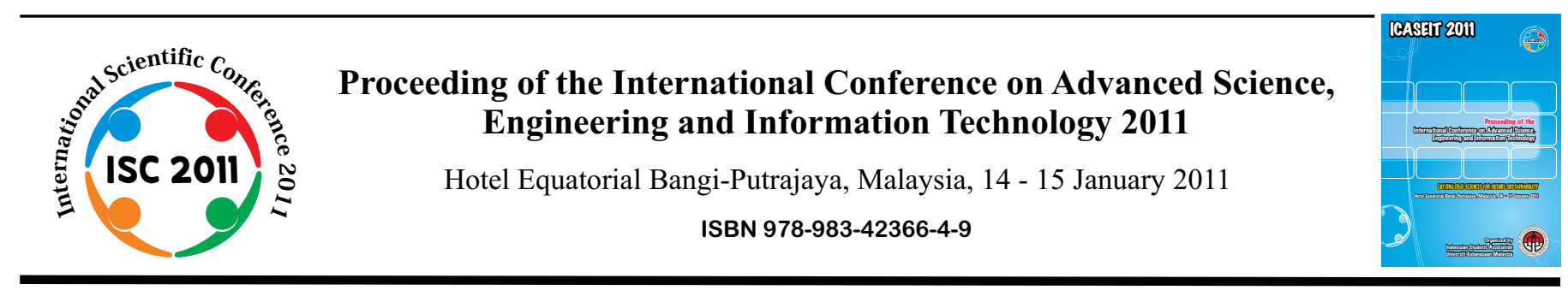

\title{
Image Colourisation for Early Infarct Detection
}

\author{
Ong Ming Kuang", Sim Kok Swee\#, Chia Fu Keong\#, Tan Chin Kang", Ng Jia Tsing", Tan Tiong Lang\#, \\ Tso Chih Ping*, Choo Sad Leng+, Rozalina Abd. Hamid+ \\ ${ }^{\#}$ Faculty of Engineering and Technology, Multimedia University, Melaka, Malaysia \\ FET, Multimedia University, 75450, Melaka, Malaysia, \\ Tel.:+606-2523480, E-mail: kssimemmu. edu.my \\ *School of MAE, Nanyang Technological University, Singapore \\ +Department of Diagnostic Imaging, Hospital Melaka, Malaysia
}

\begin{abstract}
Although early detection of infarct sign can provide early treatment to patients, early infarct detection on brain images is well-known to be difficult due to their subtle signs. A method is proposed to aid the detection of infarct through image colourisation based on Hounsfield units. A test was conducted in a private university to evaluate the algorithm, and the method appeared helpful and robust. The limited results showed that students with and without medical background had improved their ability of detecting early sign by $5.5 \%$ with the help of image colourisation.
\end{abstract}

Keywords - Colour, Early infarct, Hounsfield units, Stroke Detection

\section{INTRODUCTION}

Brain lesion can be defined as an area of damage brain tissue due to injury or disease, and can be caused by several ways, particularly infection in the brain, abnormal grow of clusters veins, head injuries, Alzheimer's disease, multiple sclerosis and also prominent cerebral infarct or stroke [1]. According to the statistics from World Health Organization (WHO), in 2007, 15 million people suffered from stroke globally. Out of these, stroke claimed the life of 5 million, and another 5 million were permanently disabled [2].

Stroke is the third among the reasons for acute death [3]. It is also the first among the reasons for neurological dysfunction [3]. There are mainly two types of stroke, namely ischemic stroke and hemorrhagic stroke as shown in Fig. 1 [2]. The occurrence of Ischemic stroke is due to sudden blockage of the arteries in the brain. The blockage causes the lack of oxygen in the blood [2]. As a result, the brain cells die. On the contrary, hemorrhagic stroke is due to sudden rupture of an artery, causing the blood flowing into other brain tissue [2].

At present, CT scans and MRI are the two brain imaging techniques mostly used in hospital to examine the brain lesion. These two techniques can effectively disclose different types of brain tissues. With the advanced technology, the images for these two imaging modalities are in DICOM format which include corresponding patient data as well as the images [4]. However, CT scan is still the prefer choice than MRI due to lower cost and wider availability [4] [5]. In addition, CT imaging can provide information especially in the acute stage.

Previous works had already been proposed and implemented to detect the lesion in brain images. Chawla et al. proposed an automatic detection and classification of stroke from brain CT images [6]. The proposed method consists of three steps, namely image enhancement, midline symmetry detection and abnormal classification for brain scan images [6]. In the classification process, two level classification methods are used to detect 
brain abnormalities by using characteristics in the intensity and wavelet domain [6]. Meanwhile, Diyana et al. identified brain abnormalities by using symmetrical features [7]. Any tilted brain image is rotated correctly before symmetrical axis is determined [7]. After that, the area and centroid of the abnormalities are chosen as the features in the rule-based abnormalities detection [7]. A new method of detecting the midline shift in brain injury was also proposed by Liu et al. [8]. The forming of midline is predicted from the brain lesion by using Linear regression model (H- MLS model) [8]. Hara et al. proposed another way to detect the midline based on the skull contour of the brain [9].

Most of the existing works on stroke detection mainly focus on hemorrhagic stroke without paying much attention on ischemic stroke. In this project, early infarct detection through image colourisation is proposed to aid the radiologists in providing diagnosis to the patient. The proposed method consists of two main parts, which are image contrast stretching and image colourisation. All of the method details will be discussed in the following section.

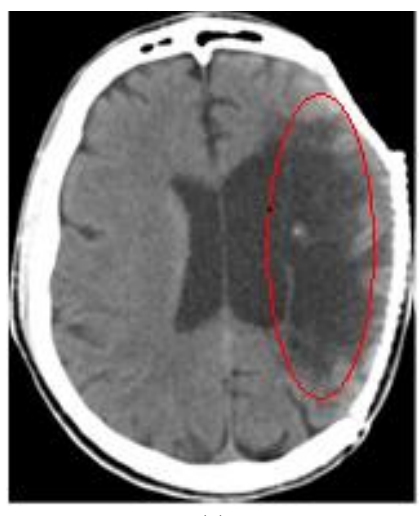

(a)

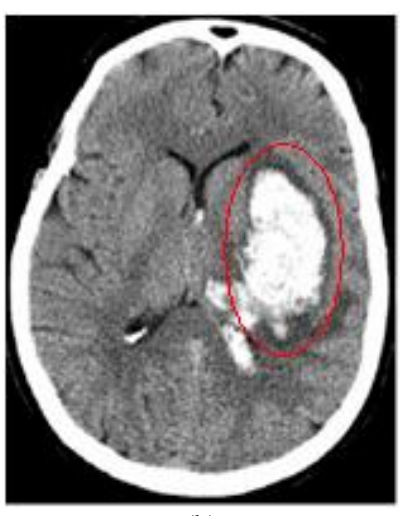

(b)
Fig. 1: Two types of stroke as indicated by red circle: (a) Ischemic stroke and (b) Hemorrhagic stroke [6]

\section{PROPOSED METHOD}

\section{A. Image Contrast Stretching}

With the advanced technology, all brain images are in DICOM format. The range of the brain image pixel is very large, corresponding to 16-bit or 65536 greyscale levels. The huge range can cause the unperceived information when the brain image is viewed without any windowing as shown in Fig. 2(a). However, most of the important brain structures are within the range of 0 to 80 in
Hounsfield unit (HU). Consequently, windowing process has to be performed. Firstly, initial brain image in DICOM format has to be adjusted before conversion to greyscale by using the equation 1 . The rescale slope and rescale intercept value can be obtained from the metadata of DICOM image. This is to ensure that all DICOM image can be converted to greyscale image properly regardless of different rescale intercept on different machines.

$$
\operatorname{Img}=\operatorname{Im} X \text { rescale slope }+ \text { rescale intercept },
$$

where $\mathrm{Im}$ is the original pixel value in DICOM format, and Img is the new pixel value in DICOM format.

After that, the new HU represented by Img is converted to 8-bit greyscale value as shown in equation 2. This windowing will result in an image displaying HU from 0 to 80 only, while the remaining $\mathrm{HU}$ are represented by black or white colour. The windowed image is then converted to the range from 0 (black) to 255 (white). The window center (WinCenter) is set to 40 while the window width (WinWidth) is set to 80 . The final greyscale image is produced and shown in Fig. 2(b).

$$
\text { grey img }=255 \mathrm{x} \frac{\text { Img }- \text { WinMin }}{\text { WinMax }- \text { WinMin }},
$$

$$
\begin{aligned}
& \text { where WinMax }=\text { WinCenter }+\frac{\text { WinWidth }}{2} \\
& \text { and } \quad \text { WinMin }=\text { WinCenter }-\frac{\text { WinWidth }}{2}
\end{aligned}
$$

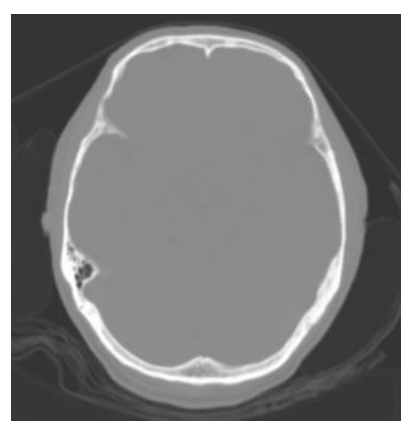

(a)

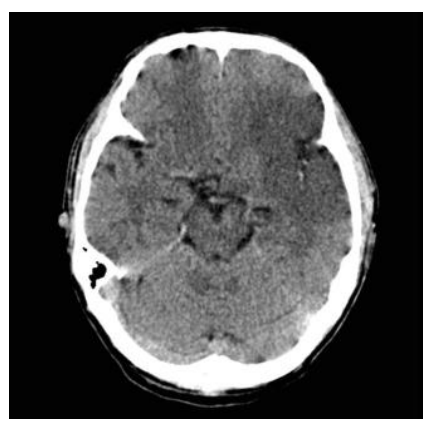

(b)
Fig. 2: A CT brain image of early infarct case: (a) image before windowing and (b) greyscale image after conversion 


\section{B. Image Colourisation}

After converting brain image from DICOM format to greyscale, the image is then coloured. Different colours are assigned for each range of greyscale values to improve the visualization of different types of brain structures. The greyscale level can be obtained in Table 1 based on the HU for brain structures. A colour map is chosen to apply the colourisation on the brain image as shown in Fig. 3. Three basic colours are chosen, which are red, blue and yellow that form a colour wheel. Among these three colours, red colour has always been considered to be the most contrasting and prominent colour. Therefore, it is chosen for the greyscale level of 64 to 96 which covers the stroke tissue. Among the blue and yellow colour, gray matter is represented by yellow colour for brighter appearance in greyscale brain image. Lastly, blue colour is chosen to cover the greyscale levels of 96 to 128. In addition, the greyscale level that falls below 64 are black, while those above 192 are white in colour. Fig. 3 shows the new colour map values to represent the greyscale levels from 0 to 255 for a CT brain image.

TABLE 1: THE RANGE OF GREYSCALE LEVELS FOR IMPORTANT BRAIN STRUCTURES

\begin{tabular}{|c|c|c|}
\hline Brain Structure & Hounsfield unit range & Greyscale level \\
\hline Stroke tissues & $20-30$ & $64-96$ \\
\hline White Matter & $30-40$ & $96-128$ \\
\hline Gray Matter & $50-60$ & $160-192$ \\
\hline
\end{tabular}

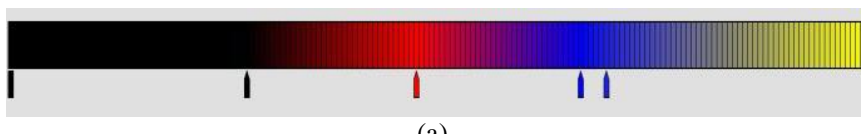

(a)

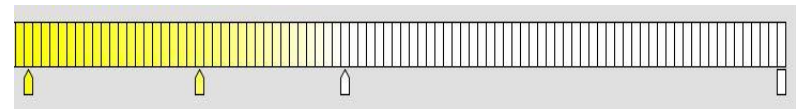

(b)

Fig. 3: (a) New colour map of 255 levels for brain image colourisation and (b) the continuation of (a)

With the proposed colour map, the visibility of early infarct area is greatly enhanced, where the stroke tissues is represented by red colour. Besides, the attention of medical practitioners is attracted to provide diagnosis if a big area of red colour appears in the brain image. However, sign of early infarct detection does not merely lie on spotting for red colour, but rather the comparison of the colour in brain symmetry. Chronic or old infarcts will be even more obvious through black colour representation. It is very hard to miss a chronic infarct in greyscale brain image. However, the infarct is more prominent with the aid of colours. Fig. 4 shows the early infarct area.

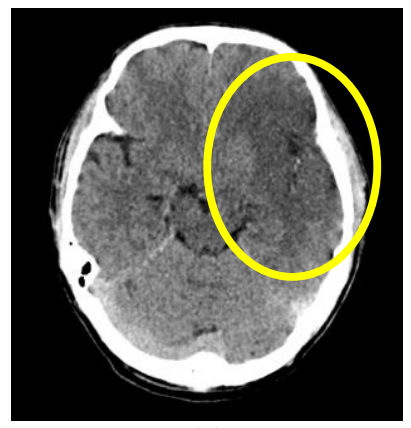

(a)

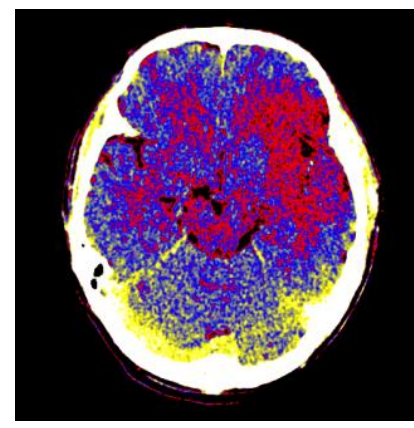

(b)
Fig. 4: A CT brain image of early infarct case: (a) the early infarct area indicated by yellow circle and (b) the early infarct area represented by red colour

\section{RESULTS AND DISCUSSIONS}

In this project, a group of candidates consists of 20 undergraduate students with and without medical background had evaluated the system based on 10 patients' brain images. All results are verified by experience radiologists. The final score is calculated as:

$$
\text { score }=\frac{\text { right }}{\text { right }+ \text { wrong }+ \text { miss }} \times 100 \%
$$

Right is the condition where candidate and doctor also detect the same area. Wrong is defined as candidate detects the area but not doctor while miss is where doctor detects the area but not candidate.

Table 2 shows the scores of the evaluation without colour aid. The result shows that the proposed method improved the scores of the evaluation by $5.5 \%$ as tabulated in Table 3 . As a result, the proposed method is proved to be successful in helping the students to make better diagnosis for early infarct cases.

The improvement of score is important for those who lack experience in diagnosing early infarct cases. The proposed method helps them to improve their diagnosis. However, there is also an increase in false positives ("wrong") when the candidates over diagnose the existence of an infarct, not matching with the report from the radiologist. These false positives will reduce the accuracy of diagnosis, which brings not entirely 
harmful to a patient. This is due to the fact that some students have never seen CT scan images, and most of them are not familiar with the brain anatomy before taking the test. As a result, more training has to be conducted to achieve more accurate reading of the coloured images. More results of early infarct detection through image colourisation are shown in Fig. 5.

TABLE 2: SCORE OF STUDENTS WITHOUT AID

\begin{tabular}{|l|c|}
\hline Parameter & Students (Without aid) \\
\hline Right & 7.9 \\
\hline Wrong & 23.15 \\
\hline Miss & 19.2 \\
\hline Score & 15.72 \\
\hline
\end{tabular}

TABLE 3: SCORES OF STUDENTS WITH AID

\begin{tabular}{|l|c|}
\hline Parameter & Students (with aid) \\
\hline Right & 13 \\
\hline Wrong & 32.2 \\
\hline Miss & 16.1 \\
\hline Score & 21.2 \\
\hline
\end{tabular}

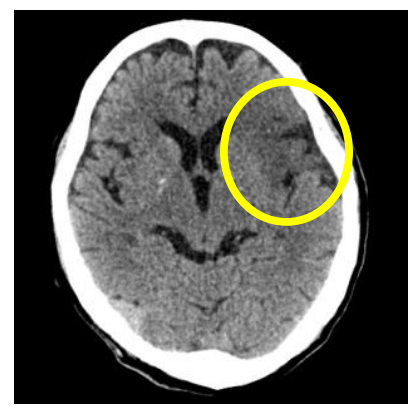

(a)

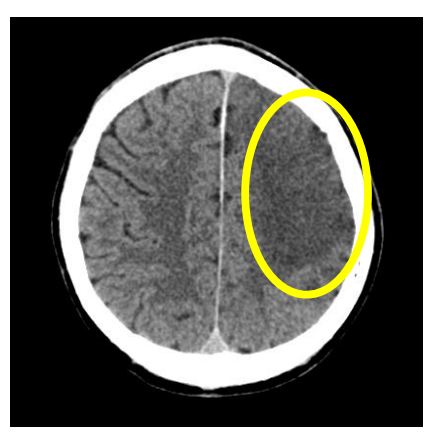

(c)

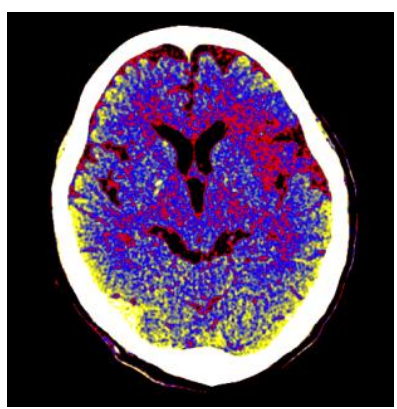

(b)

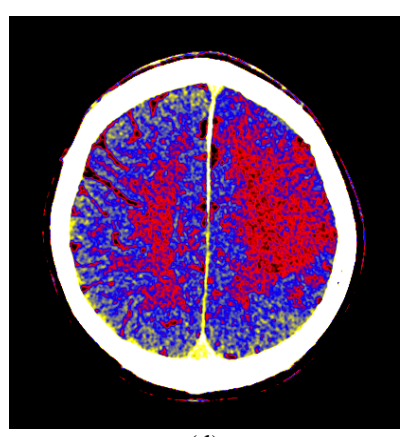

(d)
Fig. 5: CT brain images of infarct cases: (a), (c) infarct cases where early infarct is indicated by yellow circle and (b), (d) the final image colourisation.

\section{CONCLUSIONS AND FUTURE WORK}

Colourisation of CT brain images is proposed by using simple colour map based on different Hounsfield units for different brain structures. The result shows that the early infarct detection with aid is improved by $5.5 \%$. This is important and valuable for medical practitioner in providing treatment to patient at the early stage. The sample size for students with and without medical background is not more than 50 . The detection system is not fully tested and evaluated if the sample size is small. This limitation is due to the limited time frame of the study, as well as the unavailability of the students in university. In future, the sample size will be targeted to be more than 150 candidates for the evaluation, and more than 25 brain images of early infarct cases will be included in the system to enable robust system training takes place.

\section{ACKNOWLEDGMENT}

We would like to acknowledge assistance and support from Multimedia Development Corporation for us to do brain early infarct research.

\section{REFERENCES}

[1] S. E. Smith (2010), What are brain lesions? [online], Available: http://www.wisegeek.com/what-are-brain-lesions.htm

[2] The Internet Stroke Center (1997), About stroke [online], Available: http://www.strokecenter.org/patients/stats.htm

[3] A. Fhager, T. McKelvey, M. Persson, "Stroke detection using a broadband microwave antenna system", 4th European Conference on Antennas and Propagation, April 12-16, 2010, Barcelona, Spain, pp. C13P1-2S.

[4] M. Chawla, S. Sharma, J. Sivaswamy, and K. L. T., "A method for automatic detection and classification of stroke from brain CT images", Proc. Ann. Int. Conf. IEEE Eng. Med. Biol. Soc. (EMBC 09), 2009.

[5] A. Downie(2001), "Tutorial: CT in Head Trauma", [online]. Available:http://www.radiology.co.uk/srs-x/tutors/cttrauma/tutor.htm

[6] M. Chawla, S. Sharma, J. Sivaswamy, and K. L. T., "A method for automatic detection and classification of stroke from brain CT images", Proc. Ann. Int. Conf. IEEE Eng. Med. Biol. Soc. (EMBC 09), 2009.

[7] W. M. Diyana, W. Zaki, C. Kong, "Identifying abnormalities in computed tomography brain images using symmetrical features", International Conference on Electrical Engineering and Informatics, (ICEEI '09), 2009

[8] R. Liu, S. Li, C. L. Tan, B. C. Pang, C. C. T Lim, C. K. Lee, Q. Tian, Z. Zhang, "From hemorrhage to midline shift: A new method of tracing the deformed midline in traumatic brain injury ct images", 16th IEEE International Conference on Image Processing (ICIP), 2009.

[9] T. Hara, N. Matoba, X. Zhou, S. Yokoi, H. Aizawa, H. Fujita, K Sakashita, and T. Matsuoka, "Automated detection of extradural and subdural hematoma for contrast-enhanced CT Images in emergency medical care, Proc. SPIE, 2007, vol. 6514 651432(1-4). 